\title{
Terrain Model Registration for Single Cycle Instrument Placement
}

\author{
Matthew Deans, Clay Kunz, Randy Sargent, and Liam Pedersen \\ QSS Group / Autonomy and Robotics Area \\ NASA Ames Research Center \\ Mailstop 269-3, Moffett Field, CA 94035, USA \\ \{mdeans,ckunz,rsargent,lpedersen\}@ arc.nasa.gov
}

\begin{abstract}
This paper presents an efficient and robust method for registration of terrain models created using stereo vision on a planetary rover. Our approach projects two surface models into a virtual depth map, rendering the models as they would be seen from a single range sensor. Correspondence is established based on which points project to the same location in the virtual range sensor. A robust norm of the deviations in observed depth is used as the objective function, and the algorithm searches for the rigid transformation which minimizes the norm. An initial coarse search is done using rover pose information from odometry and orientation sensing. A fine search is done using Levenberg-Marquardt. Our method enables a planetary rover to keep track of designated science targets as it moves, and to hand off targets from one set of stereo cameras to another. These capabilities are essential for the rover to autonomously approach a science target and place an instrument in contact in a single command cycle.
\end{abstract}

\section{INTRODUCTION}

Single cycle instrument placement (SCIP) is the single greatest autonomy need for the next generation of Mars rovers, such as the planned 2009 MSL rover mission to Mars1. The goal of SCIP is to enable a planetary rover to approach and place an instrument on a scientifically interesting point on the terrain from a distance of 10 meters[1], [2]. This must happen within one command cycle, so that after an operator selects a science target and uploads a command, the next response from the rover is the requested science measurement from the target. Single cycle instrument placement will significantly increase science return per unit of operational time over the stop and move, human-in-the-loop operation of the Sojourner and MER rovers, which each require between 3 and 5 command cycles to obtain the same data.

The first step in SCIP is the navigation of the rover to a location that places the point of interest within the workspace of an arm which carries an instrument. Uncertainty about the exact target position and accumulated rover localization errors require that the rover actively keep track of where the target is in relation to itself as navigates towards it. Once positioned, the rover evaluates

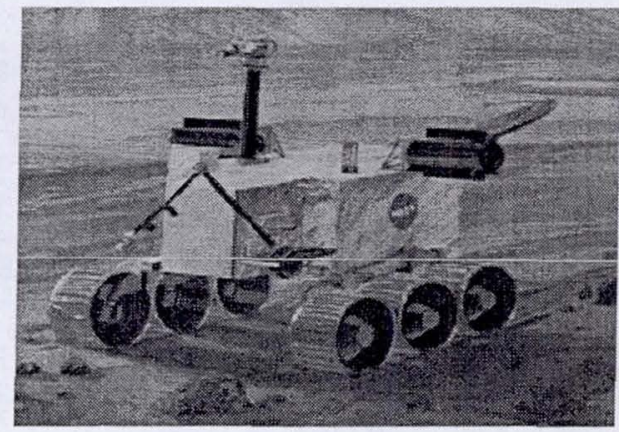

Fig. 1. Artist's conception of 2009 Mars Smart Lander [JPL]

the target to ensure the instrument can be safely placed and then moves it into place with the arm. This can require handing the target off from the cameras used to track it in the approach phase to the cameras used for close up inspection and positioning of the arm.

Terrain model registration can solve both the target tracking and target hand-off problems. Tracking is done by registering successively acquired terrain models of the target area to the initially acquired model of the target. Tracking also provides information about rover motion between views. Hand-off is done by registering the target models from two different sensors.

This paper focuses on the problem of terrain model registration. The method presented in this paper uses stereo vision to build $3 \mathrm{D}$ terrain models, then uses an algorithm similar to ICP to find the rigid transformation which aligns two models. An important difference between the method presented here and ICP is the use of a sensor model which projects the two views into a virtual range sensor. Using a rendering model removes the need to search for corresponding points with a distance heuristic. A robust error metric is then minimized, reducing the effect of outliers in the stereo models. A coarse search for the minimum is performed using a correlation based strategy which uses partial knowledge of rover motion. A fine search is performed using a general purpose robust estimation algorithm. 


\section{PREVIOUS WORK}

The Iterated Closest Point (ICP) algorithm was introduced by Chen and Medioni[3] and Besl and McKay[4] to recover a rigid transformation between two point clouds with unknown correspondence. The method relies on two steps. The first uses a nearest neighbor heuristic to establish correspondence between points. The second computes the rigid transformation between the point clouds. When only two point clouds are being aligned, the second step is computed in closed form.

A good summary of ICP and its extensions can be found in a recent survey[5]. An important extension to ICP is an objective function which uses the distance between a vertex in one model and the nearest point on the surface of the other model, rather than the nearest vertex[6]. This objective function does not penalize for motion of corresponding points along the surface.

Methods other than ICP have also been used for model registration, including the ExpectationMaximization algorithm[7], and nonlinear optimization with robust $\mathrm{M}$-estimators[8]. The latter approach is attractive for several reasons. Fitzgibbon showed that besides increasing the robustness of the solution to outliers in the data, using Levenberg-Marquardt to minimize a robust norm converges to a solution rapidly and has a significantly larger basin of attraction than least squares. For these reasons, robust estimation with LevenbergMarquardt is used in this work.

\section{APPROACH}

This section describes the technical approach used for terrain model registration. The approach relies on three key parts. The first is a sensor model which predicts the observations that should be seen under a hypothesized transformation for the surface models. The sensor model used here is a virtual range sensor, which is a reasonable approximation to the stereo system used to measure the shape of the terrain. The sensor model allows us to write an objective function which depends on the difference between what is observed and what is expected under the hypothesis.

The second part is a coarse search based on approximate knowledge of position and orientation. Assuming a fixed orientation, the virtual range sensor axes specify a coordinate frame over which a $2 \mathrm{D}$ correlation search can be performed. The coarse search finds an approximate translation which is closer to the alignment than the initial guess based on odometry.

The third part is a fine search based on LevenbergMarquardt (LM) nonlinear optimization[9], along with an extension which incorporates robust estimation using iteratively reweighted least squares (IRLS)[10], [11]. The robust optimization method is used to minimize the objective function and recover the alignment of the terrain models.

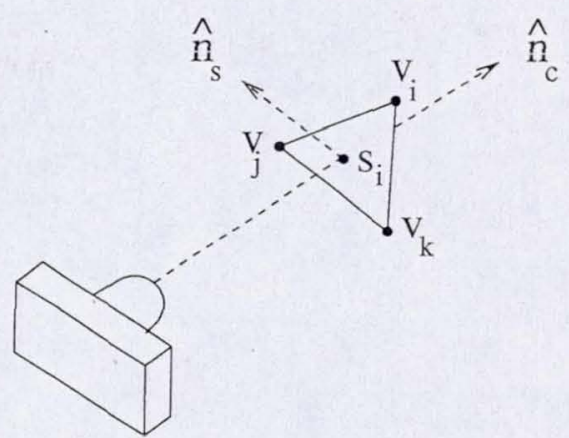

Fig. 2. Each pixel in the range image is predicted by rendering the corresponding mesh facet into a virtual range sensor.

\section{A. Sensor model}

Stereo processing results in a range image consisting of a depth estimate for every pixel in the rover stereo cameras. These depth estimates are combined to produce a 3D model of the surface. If two models of a surface are made from different locations, the rigid transformation that aligns the two models can be used to determine the coordinate transformation between views.

The surface models are represented by triangulated meshes with vertices $\mathbf{v}$ and $\mathbf{v}^{\prime}$. If the two 3D models contain some region of overlap, there is a rigid transformation that aligns the overlapping regions. The goal of registration is to find the rigid transformation that aligns the model $\mathrm{v}$ with the model $\mathrm{v}^{\prime}$. We represent the transformation using the parameter $\mathbf{p}=(x, y, z, \quad, \quad)^{T}$ corresponding to 3 translational and 3 rotational degrees of freedom. There are many ways to represent rotations; we choose Euler angles for simplicity. Singularities in the representation are not an issue since roll and pitch angles for our rover are naturally constrained to be within tolerable physical limits.

These parameter $\mathbf{p}$ defines a $4 \quad 4$ transformation matrix $\mathrm{T}_{\mathrm{p}}$. If $\mathrm{p}$ is the parameter describing the transformation between surfaces $v$ and $v^{\prime}$, then for every pair of corresponding points $\mathbf{v}_{i}$ and $\mathbf{v}_{i}^{\prime}$ the relationship

$$
\mathbf{v}_{i}^{\prime}=\mathbf{T}_{\mathrm{p}} \mathbf{v}_{i}
$$

holds. With real observations this equality will not hold exactly. The approach taken in ICP is to minimize the Euclidean distance between the corresponding points.

In this work, we project these two models into a virtual range sensor view and minimize the difference between the rendered depths at each point. The projection is done using a rendering operation which uses the hypothesized pose of a model in the camera coordinates to find the intersection of the surface of the terrain model and the rays corresponding to each pixel of the virtual range sensor. The range is then computed as the distance between the camera center and the intersection of the model surface and camera ray.

The rendering takes $O(n)$ operations, where $n$ is the number of pixels in the virtual range sensor. For each 
triangle on the mesh $\mathrm{v}^{\prime}$, the vertices $\mathrm{v}_{i}^{\prime}, \mathrm{v}_{j}^{\prime}$, and $\mathrm{v}_{k}^{\prime}$ are projected onto the image plane. For every pixel inside that triangle, the location of the intersection of the camera ray $\hat{\mathbf{n}}_{c}$ and the facet of the mesh is a point $\mathbf{s}_{i}^{\prime}$, given by

$$
\mathbf{s}_{i}^{\prime}=a_{i} \mathbf{v}_{i}^{\prime}+a_{j} \mathbf{v}_{j}^{\prime}+a_{k} \mathbf{v}_{k}^{\prime}
$$

with $a_{i}+a_{j}+a_{k}=1$. The depth to the intersection point is the length of the projection of the intersection point onto the camera ray,

$$
z_{i}=\hat{\mathbf{n}}_{c} \quad \mathrm{~s}_{i}^{\prime}
$$

The vector of all depths $z_{i}$ is denoted $z$. We fix the registration to use the coordinate frame of the surface model $v^{\prime}$ so that it does not move during registration. This means that $\mathbf{z}$ is a constant and can be computed once at the beginning of a registration.

The depth to the point $\mathbf{v}_{i}$ changes with $\mathbf{p}$. Similarly to (2) and (3), we write

$$
\mathbf{s}_{i}=\mathbf{T}_{\mathbf{p}}\left(a_{i} \mathbf{v}_{i}+a_{j} \mathbf{v}_{j}+a_{k} \mathbf{v}_{k}\right)
$$

and

$$
h_{i}(\mathbf{p})=\hat{\mathbf{n}}_{c} \quad \mathbf{s}_{i}
$$

The function $\mathbf{h}(\mathrm{p})$ is a vector containing all predicted depths. We define an objective function which is the sum of squared deviations between the projected depths

$$
J_{2}=\frac{1}{2}\left(\begin{array}{lll}
\mathbf{z} & \mathbf{h}(\mathbf{p}))^{T} \mathbf{R}^{\mathbf{1}}(\mathbf{z} \quad \mathbf{h}(\mathbf{p}))
\end{array}\right.
$$

where $\mathrm{R}$ is the measurement covariance. The use of a rendering model eliminates the need to search for corresponding points. Correspondence between points is established directly by the rendering operation since uncer the current pose hypothesis, corresponding mesh points project to corresponding range image pixels.

\section{B. Coarse registration}

We can expect our rover to have approximate knowledge of translation and rotation between observations. Dead reckoning can provide rudimentary information about both translation and rotation. On relatively short traverses, errors in dead reckoning based purely on odometry on the $\mathrm{K} 9$ rover are on the order of $10 \mathrm{~cm}$ of translation and a few degrees of rotation in yaw per meter travelied. Our rover also has sensors which measure orientation directly, including an inclinometer and a sun tracker which together fully constrain the rover orientation. These sensors are accurate to within a few degrees regardless of distance travelled.

Visual tracking methods often make use of brute-force correlation to find the 2D image plane location of a feature of interest. Searching for a 6dof rigid transformation using correlation is prohibitive, since evaluating every hypothesis on a 6D grid is expensive. However, if the orientation is approximately known, then 3 of the degrees of freedom can be eliminated, reducing the search to $3 \mathrm{dof}$. Since a virtual range image is used to evaluate each pose hypothesis, we can also eliminate the search in the camera

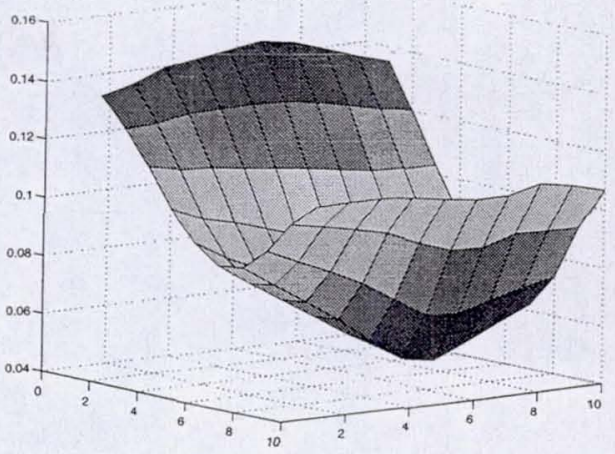

Fig. 3. Example correlation surface for coarse registration.

$z$ axis. For any translation parallel to the camera $x \quad y$ plane, the average or median $z$ deviation can be computed and removed. This reduces the $6 \mathrm{D}$ search to $2 \mathrm{D}$, making correlation feasible.

The correlation proceeds as follows. The camera location and orientation are estimated using onboard sensors, and the orientation is fixed. A grid is established in the camera sensor plane as a set of translations $x_{c}$ and $y_{c}$. The camera $z$ coordinate of each translation is zero. The approximate camera orientation is then used to rotate these translations to world coordinates $(x, y, z)$. For each translation hypothesis, the differences between corresponding pixels in the two rendered range images is computed. The median of these differences is found and subtracted out, and then the sum of absolute differences between the corrected range values is computed as a match score. The match score for each translation in the correlation grid is then interpreted as a correlation surface. The minimum value is chosen as the coarse match. An example correlation surface is shown in Figure 3.

A grid size and grid spacing must be determined over which the correlation is to be computed. Our current implementation uses an 1111 grid with a $10 \mathrm{~cm}$ spacing, which can compensate for translation errors of up to half a meter and find an initial coarse alignment that is within 5 centimeters of the solution.

\section{Fine registration}

The goal of our registration procedure is to minimize (6). LM requires the gradient and approximate Hessian

$$
\begin{aligned}
& \nabla_{\mathrm{p}} J_{2}=\underset{\mathrm{H}^{T} \mathrm{R}^{1}(\mathrm{z}}{\mathrm{h}(\mathrm{p}))} \\
& \nabla_{\mathrm{p}}^{2} J_{2} \\
& \mathrm{H}^{T} \mathrm{R}^{{ }^{1} \mathrm{H}}
\end{aligned}
$$

to compute a parameter update

$$
\mathrm{p} \leftarrow \mathrm{p}+\left(\nabla_{\mathrm{p}}^{2} J_{2}+\mathrm{I}\right){ }^{1} \nabla_{\mathrm{p}} J_{2}
$$

The parameter ensures that (8) is well conditioned and takes an appropriate step. A more complete description of the LM algorithm is beyond the scope of this paper. Several useful descriptions exist [12], [9]. However, the Jacobian of the sensor model is specific to the application described here. 


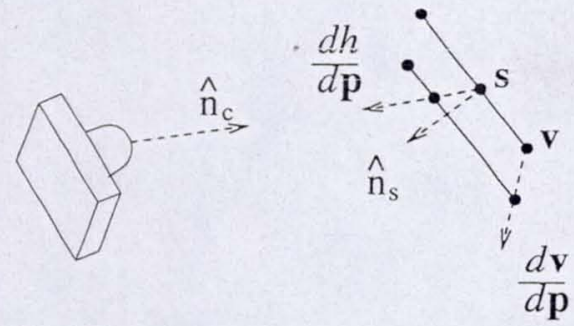

Fig. 4. The Jacobian of the depth measurement is found by projecting the derivative of the vertex locations onto the surface normal.

The Jacobian $\mathrm{H}$ is the change in the rendered depth at each point with respect to a change in the transformation parameter $\mathbf{p}$. Motion of the point $\mathrm{s}_{i}$ on a polygon can be decomposed into motion normal to the plane and motion parallel to the plane. Motion parallel to the plane does not change the depth. The depth only changes with motion normal to the plane.

The change of the point $s_{i}$ is described by $\partial s_{i} / \partial p$, which is a linear combination of the derivatives $\partial \mathbf{v} / \partial \mathbf{p}$ with the same coefficients used during rendering in (4). The projection of the derivative onto the surface normal is

$$
\frac{\partial \mathbf{s}_{i}}{\partial \mathbf{p}_{\perp}}=\hat{\mathbf{n}}_{c}\left(a_{i} \frac{\partial \mathbf{v}_{i}}{\partial \mathbf{p}}+a_{j} \frac{\partial \mathbf{v}_{j}}{\partial \mathbf{p}}+a_{k} \frac{\partial \mathbf{v}_{k}}{\partial \mathbf{p}}\right)
$$

The change in depth $h_{i}$ lies along the camera normal. Its projection onto the surface normal is

$$
\frac{\partial h_{i}}{\partial \mathbf{p}_{\perp}}=\hat{\mathbf{n}}_{c} \quad \hat{\mathbf{n}}_{s} \frac{\partial h_{i}}{\partial \mathbf{p}}
$$

Equating the projections (9) and (10) we find

$$
\frac{\partial h_{i}}{\partial \mathbf{p}}=\frac{\hat{\mathbf{n}}_{c}}{\hat{\mathbf{n}}_{c} \hat{\mathbf{n}}_{s}}\left(a_{i} \frac{\partial \mathbf{v}_{i}}{\partial \mathbf{p}}+a_{j} \frac{\partial \mathbf{v}_{j}}{\partial \mathbf{p}}+a_{k} \frac{\partial \mathbf{v}_{k}}{\partial \mathbf{p}}\right)
$$

The Jacobian $\mathrm{H}$ is the matrix containing all of the gradients $\partial h_{i} / \partial \mathrm{p}$.

\section{Robust Estimation}

The $L_{2}$ norm is optimal when the observation noise is Gaussian. However, the $L_{2}$ norm may exhibit problems when it is not. For data which contains outliers, there are a family of norms () which are robust to large deviations. These are functions which have a bounded derivative far from zero, so that large deviations provide only a small contribution to the gradient of the objective function. The objective function used in this work uses the Huber norm[11],

$$
(x)= \begin{cases}c^{2}(1 \quad \cos (x / c)) & \text { if }|x| / c</ 2 \\ c|x|+c^{2}(1 / 2) & \text { if }|x| / c \quad / 2\end{cases}
$$

shown in Figure 5. When the deviation is close to zero, the Huber norm behaves similarly to the $L_{2}$ norm. When the deviation is large, the norm behaves similarly to $L_{1}$. This norm has been shown to perform well for ICP[8]. Using the robust norm, we rewrite (6) as

$$
J_{H}(\mathbf{p})=\frac{1}{2} \sum_{i}\left(z_{i} \quad h_{i}(\mathbf{p})\right)
$$

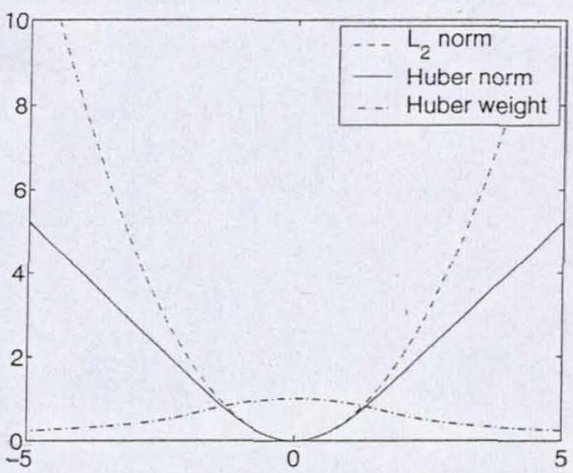

Fig. 5. Comparison of the $L_{2}$ norm and the Huber robust norm used in this work, and the weight function for weighted least squares.

and the derivatives as

$$
\begin{aligned}
& \nabla_{\mathrm{p}} J_{H}=\mathrm{H}^{T} \quad \mathrm{R}^{1}(\mathrm{z} \quad \mathrm{h}(\mathrm{p})) \\
& \nabla_{\mathrm{p}}^{2} J_{H}=\mathrm{H}^{T} \quad \mathrm{R}{ }^{1} \mathrm{H}
\end{aligned}
$$

where is a diagonal matrix of weights

$$
\omega_{i i}=w\left(z_{i} \quad h_{i}(\mathbf{p})\right)
$$

The weight function for the Huber norm is

$$
w(x)= \begin{cases}c / x \sin (x / c) & \text { if }|x| / c</ 2 \\ c /|x| & \text { if }|x| / c \quad / 2\end{cases}
$$

with $c=1.2107$. The weights are recomputed during each iteration of Levenberg-Marquardt, resulting in an iteratively reweighted least squares algorithm.

\section{EXPERIMENTAL RESULTS}

To empirically validate the performance of the registration for instrument placement, we tested our algorithm with a 4 meter traverse in the laboratory. A stereo image pair was captured using the navigation cameras on the mast of the K9 rover. A 3D model was computed and presented to an operator in the Viz visualization tool. The user specified a goal point on a rock. The selected instrument placement goal location is marked with a "+" in the left camera image shown in Figure 8. The rover moved 4 meters, stopping every meter to align the 3D model of the current view of the goal location with the 3D model created from the initial view. At a distance of 2 meters, the view switched from the navigation cameras on the mast to the hazard cameras on the underside of the rover chassis in order to provide a better view of the goal. This was accomplished using our target handoff by aligning views from the two different camera pairs.

Figure 6 shows two 3D models. The red model is the initial 3D model computed using stereo vision from a distance of 4 meters. The arrow indicates the goal location selected by the rover operator. The textured model is the final view of the rock from the hazard cameras at a distance of $50 \mathrm{~cm}$. The misregistration is a result of errors in by dead reckoning, rover kinematics, pan tilt unit calibration, etc. Figure 7 shows the result of aligning the 


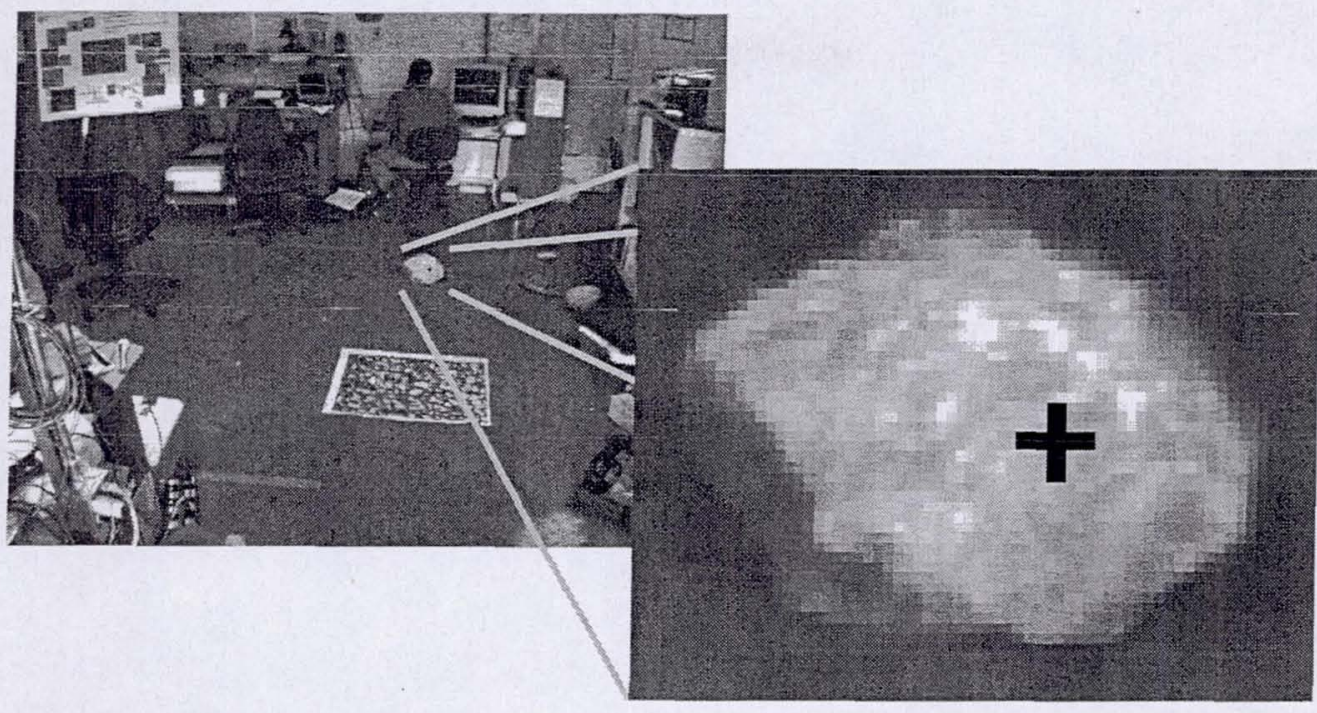

Fig. 8. Selected goal location

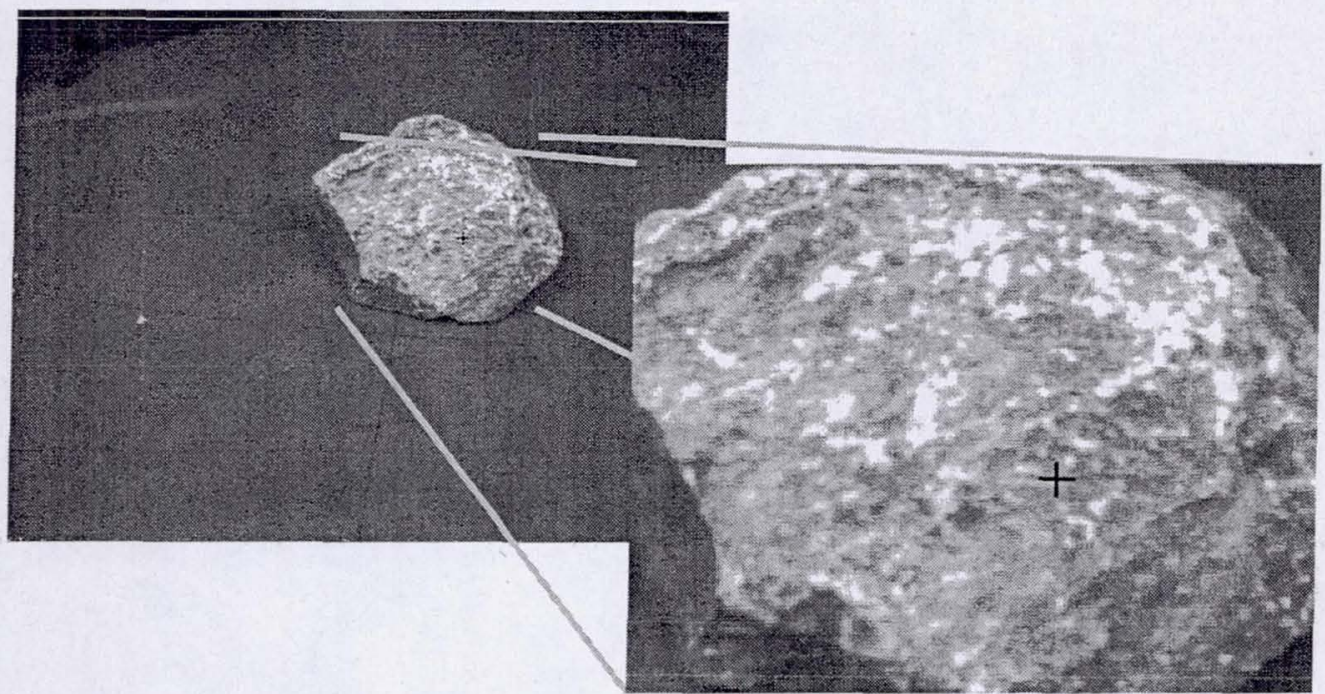

Fig. 9. Estimated goal location after registration

initial model from the navigation cameras with the final model from the hazard cameras.

The goal location on the rock can be recovered directly using the transformation which aligns the views, which is represented with an arrow in Figure 7 . The final camera view of the goal is in Figure 9, with the estimated goal location indicated with a "+". This is the intended location for the instrument, which is placed using the algorithms described in[1].

\section{DISCUSSION}

Registration of 3D surface models is an attractive method for localization and target approach. As long as the lighting conditions permit the acquisition of images for stereo, the surface models and resulting registration results are independent of the lighting conditions. This is attractive compared to $2 \mathrm{D}$ approaches which might have difficulty with tracking features or recognizing places when lighting conditions change. We can also achieve bounded error in pose estimation with respect to the target location since the initial target model can be used as long as the target remains in view.

Furthermore, $2 \mathrm{D}$ visual tracking requires the rover to spend computational effort on computations that it may be doing only for the purpose of visual pose estimation. However, NASA's current plans call for stereo vision to be used for hazard avoidance on MER in 2003 and probably on MSL in 2009. Registering the 3D models that are already created for local path planning and obstacle avoidance makes dual use of data that is being generated anyway. The marginal computation for registration is less than the computation required for building the $3 \mathrm{D}$ models 


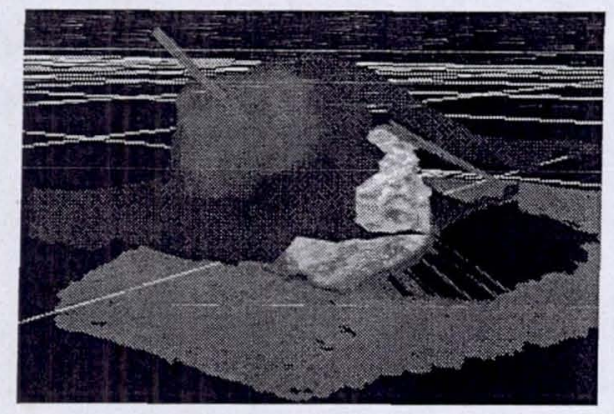

Fig. 6. Terrain models before registration.

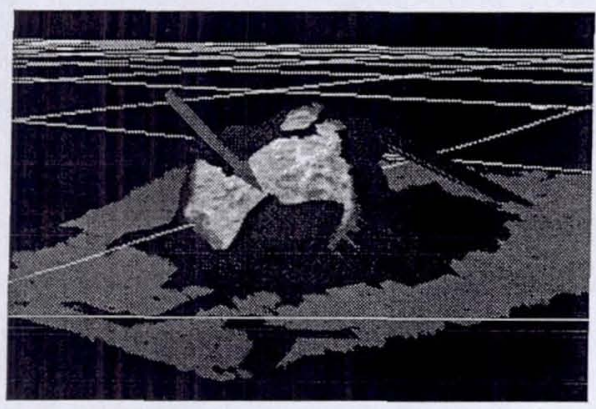

Fig. 7. Terrain models after registration.

in the first place, so most of the computational work is already done.

The robust estimation method used in this paper works quite well. The surface models used in the examples here were not regularized, resampled, or "cleaned" in any way and the results are still promising. Other reported approaches require mesh regularization and cleaning in order to ensure that meshes have similar resolutions and there are no outliers before minimizing a norm which is sensitive to large deviations. These steps may improve the results we can acheive using robust estimation but empirically are not required for it to work.

Algorithmically, our technique compares well to ICP. The rendering operation takes $O(n)$ where $n$ is the number of pixels in the virtual range image. The resolution of the virtual range image can be changed to speed up the algorithm with a corresponding loss in performance due to lack of detail in the models. Levenberg-Marquardt updates require $O(n)$ to construct and multiply matrices, but the computation of the update to the parameter is constant time since the number of dimensions in the parameter vector is fixed at 6 . In terms of convergence, the approaches have similar properties since each converges to a local minimum and will find the global optimum if the initial guess is within the basin of attraction. We have not yet done experiments to determine what that basin might look like for the different methods, but we have empirically noticed that the basin of attraction is larger for the robust norm than for least squares. We are working on a more thorough empirical comparison of our technique to ICP, and in the mean time we have also made our $3 \mathrm{D}$ terrain data public for interested readers to use for comparison with other techniques[13].

We are currently working to further extend this work. Algorithmically we are investigating ways to optimize the implementation, perhaps making use of some efficient rendering techniques. We would also like to extend this to multiview registration in order to handle more than two views at a time.

This method is being incorporated into a larger demonstration of single cycle instrument placement for improved efficiency of planetary rovers and increased science return for future Mars missions.

\section{REFERENCES}

[1] L. Pedersen, R. Sargent, M. Bualat, M. Deans, C. Kunz, S. Lee, and A. Wright. Single cycle instrument deployment for mars rovers. To appear in Proceedings of $i$-SAIRAS, 2003.

[2] M. Deans, C. Kunz, R. Sargent, and L. Pedersen. Terrain model registration for single cycle instrument placement. In To appear in Proceedings of $i$-SAIRAS, 2003.

[3] Y. Chen and G. Medioni. Object modeling by registration of multiple range images. In IEEE International Conference on Robotics and Automation, volume 3, pages 2724-2729, 1991.

[4] P. J. Besl and N. D. McKay. A method for registration of 3-d shapes. IEEE Transactions on Pattern Analysis and Machine Intelligence, 14(2):239-256, 1992.

[5] S. Rusinkiewicz and M. Levoy. Efficient variants of the icp algorithm. In Third International Conference on 3D Digital Imaging and Modeling (3DIM), 2001.

[6] P. Neugebauer. Geometrical cloning of $3 \mathrm{~d}$ objects via simuitaneous registration of multiple views. In Proceedings of the International Conference on Shape Modelling and Applications, pages 130-9, March 1997.

[7] J. Goldberger. Registration of multiple point sets using the em algorithm. In Proceedings of the International Conference on Computer Vision, volume 2, pages 730-736, 1999.

[8] A. Fitzgibbon. Robust registration of $2 \mathrm{~d}$ and $3 \mathrm{~d}$ point sets. In British Machine Vision Conference, pages 411-420, 2001.

[9] Philip E. Gill, Walter Murray, and Margaret $H$. Wright. Practical Optimization. Academic Press, 1981.

[10] P. J. Huber. Robust Statistics. John Wiley \& Sons, 1981.

[11] Zhengyou Zhang. Parameter estimation techniques: A tutorial with application to conic fitting. Technical Report No. 2676, INRIA, 1995.

[12] W. Press, S. Teukolsky, W. Vetterling, and B. Flannery. Numerical Recipes in $C$. Cambridge University Press, 1988.

[13] http://ic.arc.nasa.gov/projects/intelligentrobotics/nec/scip/data. 


\title{
Terrain model registration for single cycle instrument placement
}

\author{
Matthew C. Deans, Clay Kunz, Randy Sargent, and Liam Pedersen \\ QSS Group / Autonomy and Robotics Area \\ NASA Ames Research Center \\ Mailstop 269-3, Moffett Field, CA 94035, USA \\ \{mdeans,ckunz,rsargent,pedersen\}@arc.nasa.gov
}

\begin{abstract}
This paper presents a method for registration of terrain models created using stereo vision on a planetary rover. Most 3D model registration approaches use some variant of iterated closest point (ICP), which minimizes a norm based on the distances between corresponding points on an arbitrary $3 \mathrm{D}$ surface, where closest points are taken to be corresponding points. The approach taken here instead projects the two surface models into a common viewpoint, rendering the models as they would be seen from a single range sensor. Correspondence is established by determining which points on the two surfaces project to the same location on the virtual range sensor image plane. The norm of the deviations in observed depth at all pixels is used as the objective function, and the algorithm finds the rigid transformation which minimizes the norm. This recovered transformation can be used for visual odometry, rover pose estimation and feature hand off.
\end{abstract}

\section{Introduction}

Single cycle instrument placement (SCIP) is a critical need for the planned MSL 2009 rover mission to Mars. The goal of SCIP is to enable a planetary rover to place an instrument on a scientifically interesting point on the terrain from a distance of 10 meters with a single rover command[1].

The first step in SCIP is the navigation of the rover to a location that places the terrain point within the workspace of an arm which carries an instrument. Once positioned, the rover servos the instrument into place for taking a measurement.

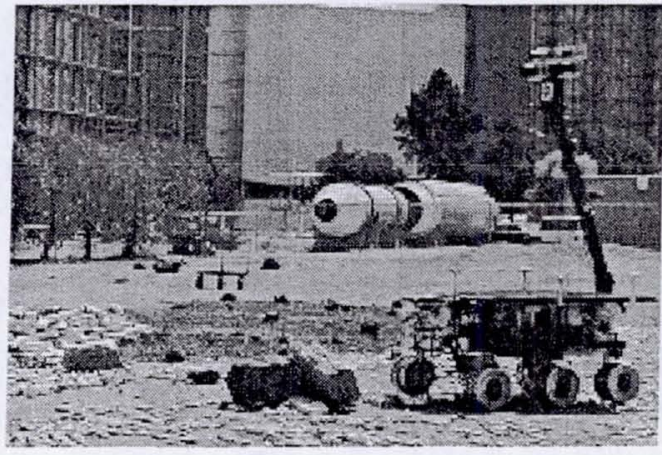

Figure 1: K9 rover placing its microscopic camera on a science target in the Marscape

All of this happens within one command cycle, so that once an operator selects a science target, the next response from the rover is the data that was requested. Single cycle instrument placement will significantly increase science return per unit of operational time over the stop and move, human-inthe-loop operation of the Sojourner rover.

Two technology needs within SCIP are pose estimation and feature hand off. Pose estimation is required in order to place the rover within reach of the science target. Registration of terrain models from different rover locations can be used to recover the pose of the rover in the different views. Feature hand off may be required to provide a better 3D model of the instrument goal location for the final step of instrument placement using imagery from hazard avoidance cameras closer to the ground. Registration of terrain models from the navigation cameras and hazard cameras provides a calibration which can be used for handing off the goal location from one stereo pair to the other. 
This paper focuses on the problem of terrain model registration. The method presented in this paper uses stereo vision to build 3D terrain models, then uses an algorithm similar to ICP to find the rigid transformation which aligns two terrain models. The important difference between the method presented here and the popular ICP algorithm is the use of a sensor model which projects the two views into a virtual range sensor and minimizes the difference between the measured depth to points on the two models. Using a rendering model removes the need to search for corresponding points with a distance heuristic. At the correct registration, corresponding points project onto the same range sensor pixel.

\section{Previous work}

The Iterated Closest Point algorithm (ICP) algorithm was proposed independently by Chen and Medioni[2] and by Besl and MacKay[3] to recover a rigid transformation between two point clouds with unknown correspondence. The method relied on a step which used a nearest neighbor heuristic to establish correspondence between points, followed by a step which computed the rigid transformation between the point clouds in closed form using techniques which had been studied earlier $[4,5]$. ICP was later extended to handle multiple views $[6,7$, $8,9]$, where there is no longer a known closed form solution for the alignment of all views even when the correspondences are known. A good survey of efficient variants on the original ICP algorithm can be found in [10].

An important extension to the original ICP algorithm was a modification to the objective function which minimizes the distance between a vertex in one model and the nearest point on the surface of the other model, rather than the nearest vertex[11]. This extension captures the fact that changes in the rigid transformation which cause a point to move along a surface should not be penalized. The derivatives of the cost function reflect the fact that the distance between a point and a plane only changes when the point moves in a direction parallel to the surface normal.

Methods other than ICP have also been used for model registration, including the general purpose Expectation-Maximization algorithm[12], and gen- eral purpose nonlinear optimization using robust M-estimators[13]. The latter approach is attractive. Fitzgibbon showed that besides increasing the robustness of the registration solution to outliers in the data, using Levenberg-Marquardt to minimize a robust norm converges to a solution rapidly and has a significantly larger basin of attraction than least squares. Because of these properties, robust estimation with Levenberg-Marquardt is used in this work.

\section{Approach}

This section describes the technical approach used for terrain model registration. The approach relies on two key parts. The first is a sensor model which predicts the observations that should be seen under a hypothesized transformation for the surface models. The second is the Levenberg-Marquardt[17, 18, 15] nonlinear optimization method, along with an extension which incorporates robust estimation using iteratively reweighted least squares[16, 19, 20].

\subsection{Sensor modeling}

For every pixel in the left camera image for which a correspondence is found in the right camera image, the stereo algorithm estimates the depth to that point. These depth estimates are combined to produce a 3D model of the surface. If two models of a surface are made from different locations, the rigid transformation that aligns the two models can be used to determine the coordinate transformation between views.

The surface models are represented by triangulated meshes with vertices $\mathbf{v}$ and $\mathbf{v}^{\prime}$. If the two $3 \mathrm{D}$ models contain some region of overlap, there should be a rigid transformation that aligns the two overlapping regions on the two surfaces. We represent the rigid transformation using the parameter vector $\mathbf{p}=(x, y, z, \alpha, \beta, \gamma)^{T}$ corresponding to 3 translation directions plus rotation in roll, pitch, and yaw. These parameters define a transformation matrix $T_{\mathrm{p}}$. If $\mathrm{p}$ is the parameter describing the transformation between surfaces $\mathbf{v}$ and $\mathbf{v}^{\prime}$, then for every pair of corresponding points $\mathbf{v}_{i}$ and $\mathbf{v}_{i}^{\prime}$ the relationship

$$
\mathbf{v}_{i}^{\prime}-\mathbf{T}_{\mathbf{p}} \mathbf{v}_{i}=0
$$




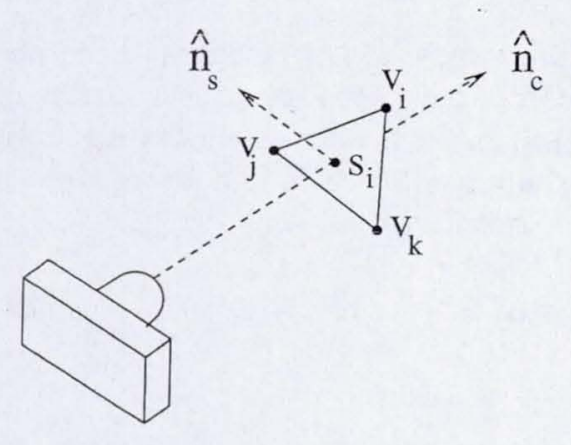

Figure 2: Each pixel in the range image is predicted by rendering the corresponding mesh facet into a virtual range sensor.

holds. With real observations this equality will not hold. The approach taken in ICP is to minimize the Euclidean distance between points. In this work, we project these two models into a virtual range sensor view and minimize the difference between the depths at each point.

Rendering takes $O(n)$ operations, where $n$ is the number of pixels in the virtual range sensor. For each facet on the mesh $\mathbf{v}^{\prime}$, the three vertices $\mathbf{v}_{i}^{\prime}, \mathbf{v}_{j}^{\prime}$, and $\mathbf{v}_{k}^{\prime}$ are projected onto the image plane, creating a triangle. For every pixel inside that triangle, the location of the intersection of the camera ray $\hat{\mathbf{n}}_{c}$ and the facet of the mesh is a point $\mathbf{s}_{i}^{\prime}$ which is a convex combination of the vertices,

$$
\mathbf{s}_{i}^{\prime}=\alpha_{i} \mathbf{v}_{i}^{\prime}+\alpha_{j} \mathbf{v}_{j}^{\prime}+\alpha_{k} \mathbf{v}_{k}^{\prime}
$$

with $\alpha_{i}+\alpha_{j}+\alpha_{k}=1$. The depth to the intersection point is the $z$ coordinate in the camera frame,

$$
z_{i}=\hat{\mathbf{n}}_{c} \cdot \mathbf{s}_{i}^{\prime}
$$

The vector of all depths $z_{i}$ is denoted $\mathbf{z}$. The surface model $\mathbf{v}^{\prime}$ does not move during registration, so $\mathbf{z}$ is a constant.

The depth to the point $\mathbf{v}_{i}$ changes with $\mathbf{p}$. Similarly to (2) and (3), we write

$$
\begin{aligned}
\mathbf{s}_{i} & =\mathbf{T}_{\mathbf{p}}\left(\alpha_{i} \mathbf{v}_{i}+\alpha_{j} \mathbf{v}_{j}+\alpha_{k} \mathbf{v}_{k}\right) \\
h_{i}(\mathbf{p}) & =\hat{\mathbf{n}}_{c} \cdot \mathbf{s}_{i}
\end{aligned}
$$

and $\mathbf{h}(\mathbf{p})$ is the vector containing all predicted depths $h_{i}(\mathbf{p})$. We then define an objective function which is the sum of squared deviations between the projected depths

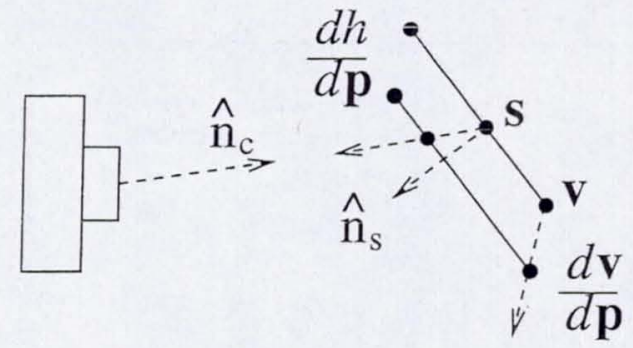

Figure 3: The Jacobian of the depth measurement is found by projecting the derivative of the vertex locations onto the surface normal.

$$
J_{2}=\frac{1}{2}(\mathbf{z}-\mathbf{h}(\mathbf{p}))^{T} \mathbf{R}^{-1}(\mathbf{z}-\mathbf{h}(\mathbf{p}))
$$

The goal of the registration then is to minimize (5). Using Levenberg-Marquardt requires the gradient and approximate Hessian

$$
\begin{aligned}
\nabla_{\mathrm{p}} J_{2} & =-\mathbf{H}^{T} \mathbf{R}^{-1}(\mathrm{z}-\mathrm{h}(\mathrm{p})) \\
\nabla_{\mathrm{p}}^{2} J_{2} & \approx \mathrm{H}^{T} \mathbf{R}^{-1} \mathbf{H}
\end{aligned}
$$

to compute the updated parameter

$$
\mathbf{p}^{(i+1)}=\mathbf{p}^{(i)}+\left(\nabla_{\mathbf{p}}^{2} J_{2}+\lambda \mathbf{I}\right)^{-1} \nabla_{\mathbf{p}} J_{2}
$$

The parameter $\lambda$ is used to dynamically $\operatorname{mix}$ Newton-Raphson style updates with gradient descent style updates. If an update results in $J\left(\mathbf{p}^{(i+1)}\right)>J\left(\mathbf{p}^{(i)}\right)$ then $\lambda$ is increased and $\mathbf{p}^{(i+1)}$ recomputed. A more complete description of the general use of Levenberg-Marquardt optimization is beyond the scope of this paper. Several useful descriptions exist[14, 15]. However, the Jacobian $\mathbf{H}=\frac{\partial \mathbf{h}}{\partial \mathbf{p}}$ warrants some explanation.

The Jacobian $\mathbf{H}$ is the change in the rendered depth at each point given a change in the transformation parameter $\mathbf{p}$. Any motion of the polygon on which the point $\mathbf{s}_{i}$ lies can be decomposed into motion normal to the plane and motion parallel to the plane. Motions parallel to the plane do not change the depth to the point. The depth only changes with motion normal to the plane.

The change of the point $\mathbf{s}_{i}$ is described by $\partial \mathbf{s}_{i} / \partial \mathbf{p}$, which is a linear combination of the derivatives $\partial v / \partial p$ with the same coefficients used during rendering in (4). The projection of the derivative onto the surface normal is

$$
\frac{\partial \mathbf{s}_{i}}{\partial \mathbf{p}_{\perp}}=\hat{\mathbf{n}}_{c} \cdot\left(\alpha_{i} \frac{\partial \mathbf{v}_{i}}{\partial \mathbf{p}}+\alpha_{j} \frac{\partial \mathbf{v}_{j}}{\partial \mathbf{p}}+\alpha_{k} \frac{\partial \mathbf{v}_{k}}{\partial \mathbf{p}}\right)
$$




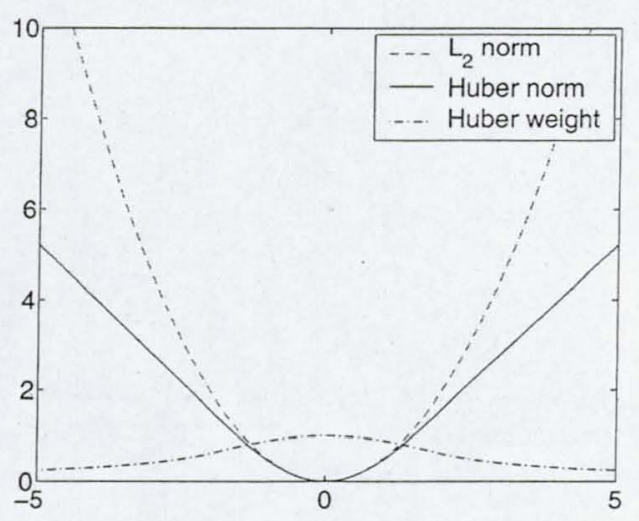

Figure 4: Comparison of the $L_{2}$ norm and the Huber robust kernel used in this work, and the weight function for weighted least squares.

The change in depth $h_{i}$ lies along the camera normal. Its projection onto the surface normal is

$$
\frac{\partial h_{i}}{\partial \mathbf{p}} \perp=\hat{\mathbf{n}}_{c} \cdot \hat{\mathbf{n}}_{s} \frac{\partial h_{i}}{\partial \mathbf{p}}
$$

Equating the projections (8) and (9) we find

$$
\frac{\partial h_{i}}{\partial \mathbf{p}}=\frac{1}{\hat{\mathbf{n}}_{c} \cdot \hat{\mathbf{n}}_{s}} \hat{\mathbf{n}}_{c} \cdot\left(\alpha_{i} \frac{\partial \mathbf{v}_{i}}{\partial \mathbf{p}}+\alpha_{j} \frac{\partial \mathbf{v}_{j}}{\partial \mathbf{p}}+\alpha_{k} \frac{\partial \mathbf{v}_{k}}{\partial \mathbf{p}}\right)
$$

The Jacobian $\mathbf{H}$ is the matrix containing all of the gradients $\partial h_{i} / \partial \mathbf{p}$.

\subsection{Robust Estimation}

The $L_{2}$ norm is optimal when the observation noise is Gaussian. However, the $L_{2}$ norm is known to exhibit problems when the data contains outliers. For data which may contain outliers, there are a family of norms $\rho()$ which are robust to large deviations. These are functions which have a unique minimum at zero and a bounded derivative far from zero, so that large deviations provide only a small contribution to the gradient of the objective function. The kernel used in this work is the Huber norm[20],

$$
\rho(x)= \begin{cases}c^{2}(1-\cos (x / c)) & \text { if }|x| / c<\pi / 2 \\ c|x|+c^{2}(1-\pi / 2) & \text { if }|x| / c \geq \pi / 2\end{cases}
$$

shown in Figure 4. When the deviation is close to zero, the Huber norm behaves similarly to the $L_{2}$ norm. When the deviation is large, the norm behaves similarly to $L_{1}$, i.e. the derivative of the norm does not increase as the deviation increases. Robust estimators have been used for a variety of applications[16]. In particular, this norm has been shown to perform well for ICP[13]. Using the robust norm, we rewrite (5) as

$$
J_{H}(\mathbf{p})=\frac{1}{2} \sum_{i} \rho\left(z_{i}-h_{i}(\mathbf{p})\right)
$$

and the derivatives as

$$
\begin{aligned}
\nabla_{\mathbf{p}} J_{H} & =\mathrm{H}^{T} \Omega \mathrm{R}^{-1}(\mathrm{z}-\mathrm{h}(\mathrm{p})) \\
\nabla_{\mathrm{p}}^{2} J_{H} & =\mathrm{H}^{T} \Omega \mathrm{R}^{-1} \mathrm{H}
\end{aligned}
$$

where $\Omega$ is a diagonal matrix of weights

$$
\omega_{i i}=w\left(z_{i}-h_{i}(\mathbf{p})\right)
$$

The weight function is derived from the robust norm, and several examples are shown in [20]. The weight function for the norm used in this work is

$$
w(x)= \begin{cases}c / x \sin (x / c) & \text { if }|x| / c<\pi / 2 \\ c /|x| & \text { if }|x| / c \geq \pi / 2\end{cases}
$$

with $c=1.2107$. The weights are recomputed during each iteration of Levenberg-Marquardt, resulting in an iteratively reweighted least squares (IRLS) optimization.

\section{Experimental results}

To empirically validate the performance of the registration, we tested the algorithm using a few examples of 3D surface registration problems which are part of a single cycle instrument placement system. The 3D models used in these experiments are all built using stereo algorithms which run onboard the K9 rover using images from three different stereo camera pairs. Table 1 describes the stereo camera pairs used on the rover. The ter-

\begin{tabular}{|l|l|l|}
\hline Camera pair & Location & FOV \\
\hline Science cam & top of mast & narrow \\
Nav cam & top of mast & moderate \\
Haz cam & below solar panel & wide \\
\hline
\end{tabular}

Table 1: Stereo camera pairs on K9 rover rain models derived from stereo imaging are used for a variety of purposes including visualization for 
scientists and rover operators as well as onboard autonomous hazard avoidance. Registration of 3D models can also enable the applications outlined in Table 2. Examples of these are shown below.

\begin{tabular}{|l|l|l|}
\hline Application & Camera pair & Camera pair \\
\hline Visual odometry & Nav cam & Nav cam \\
Pose estimation & Nav cam & Science cam \\
Target hand off & Haz cam & Nav cam \\
\hline
\end{tabular}

Table 2: Applications of 3D registration and the stereo pairs used for each.

\subsection{Visual odometry}

Visual odometry can be achieved by registration of 3D surface models created using images from the navigation cameras before and after the rover moves. The transformation which aligns the views is directly related to the change in pose of the rover, leading to an odometry measurement between poses. For the visual odometry experiment the rover was placed in the Marscape and incrementally commanded to move forward one meter or turn $45^{\circ}$. The 3D models acquired before and after each move were registered using dead reckoning as an initial guess for the alignment.

Figure 5 shows the differences in depth between the rendered surfaces using the transformation from dead reckoning, before the registration algorithm is applied. Medium gray pixels denote zero error, while white and black denote errors of more than $10 \mathrm{~cm}$. Figure 6 shows the depth errors found after optimizing the $L_{2}$ norm in (5) using Levenberg-Marquardt. While the ground plane is well aligned, there are large errors where a rock in the foreground is misaligned. Figure 7 shows the result of optimizing the robust norm in (12). By minimizing the robust norm the algorithm is able to correct the errors seen in Figure 6. This is consistent with Fitzgibbon's results and the claim that the robust norm has a wider basin of attraction than $L_{2}[13]$.

Figure 8 shows the convergence of the robust norm through time for several runs. Typically, convergence is achieved within 5-10 iterations. The two convergence curves which show 20 and 38 iterations correspond to point turns, where the overlap in the models is poor.

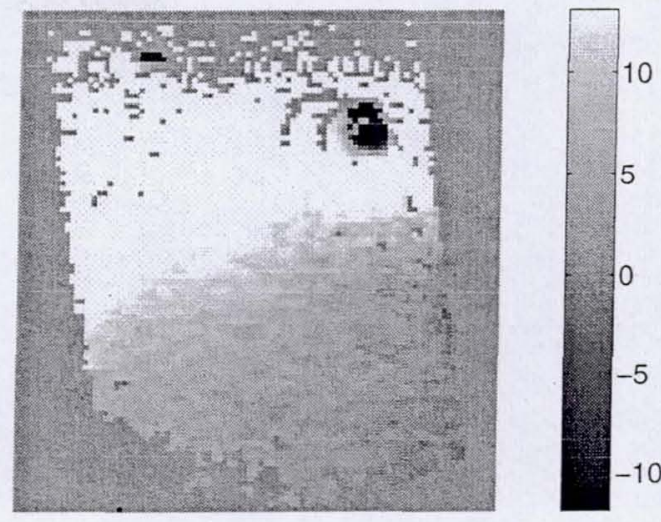

Figure 5: Initial depth errors for starting guess provided by dead reckoning for the visual odometry example. Error measured in centimeters.

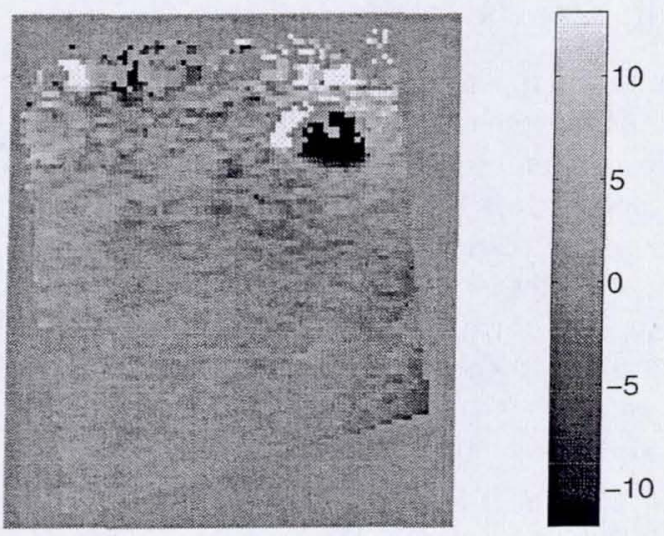

Figure 6: Final depth errors after convergence with L2 norm for the visual odometry example. Error measured in centimeters.

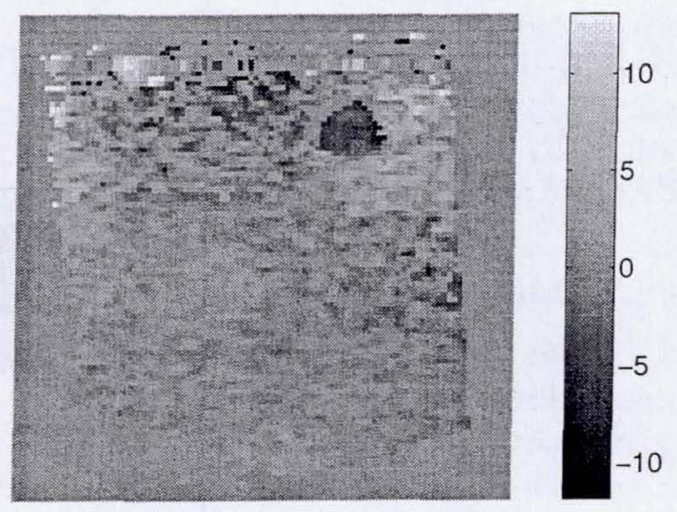

Figure 7: Final depth errors after convergence with Huber norm for the visual odometry example. Error measured in centimeters. 


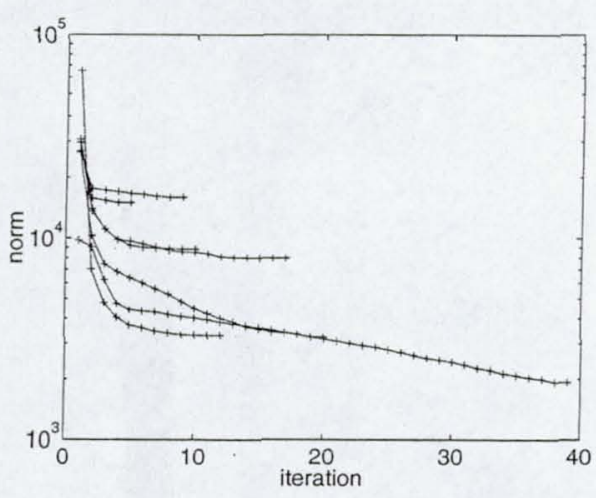

Figure 8: Convergence of the norm for several visual odometry examples

\subsection{Pose estimation}

Pose estimation may also be done relative to a map of the environment built earlier. This may be done, for example, by building an extensive 3D panoramic model using the high resolution, narrow field of view science cameras from a single location and using the model as a 3D map. The rover can repeatedly register terrain models captured by the navigation cameras against the larger more accurate model as it moves. The transformation which aligns the nav cam model to the previous map is directly related to the pose of the vehicle in the coordinate frame of the rover when the panoramic model was created. Figure 9 and 10 show an example of the registration of 3D models created by the navigation cameras and science cameras. The initial alignment again comes from dead reckoning, but once the rover has localized, the next pose can be initialized using the previous registration plus an incremental dead reckoning estimate. Figure 11 shows the initial and final depth errors for the models in Figure 9 and Figure 10.

\subsection{Feature hand off}

In the context of instrument placement it may be necessary to coordinate multiple stereo pairs. For example, for the task of placing an instrument against a rock in front of the rover, the Haz cams might have a better vantage point to image the goal location when the rover is close to a rock which was selected by rover operators using the navigation cameras. By registering the 3D models from

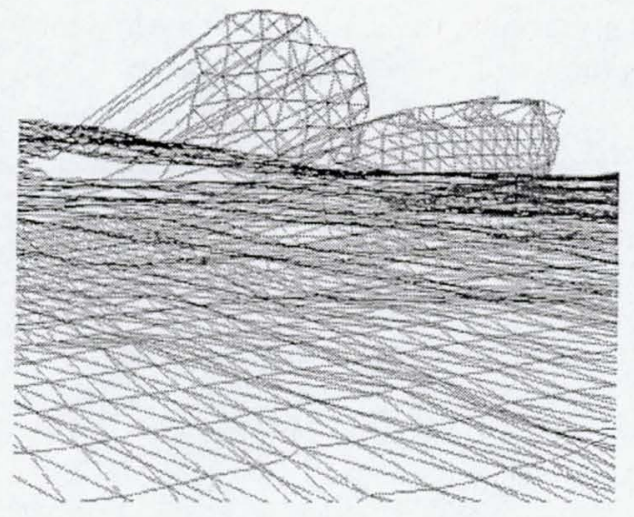

Figure 9: Alignment before registration for the pose estimation example.

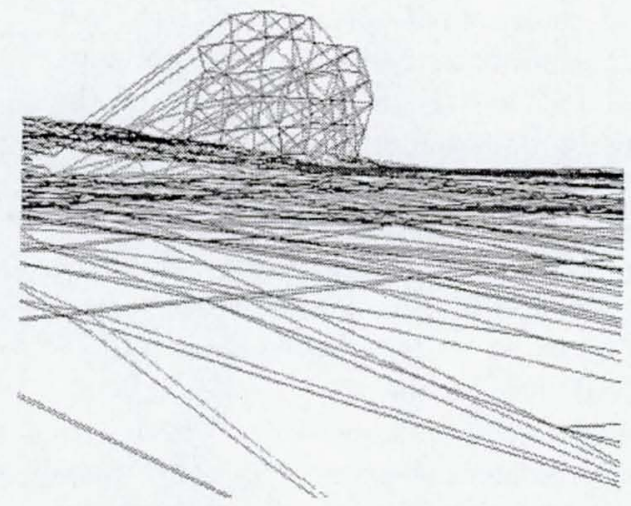

Figure 10: Alignment after registration for the pose estimation example.
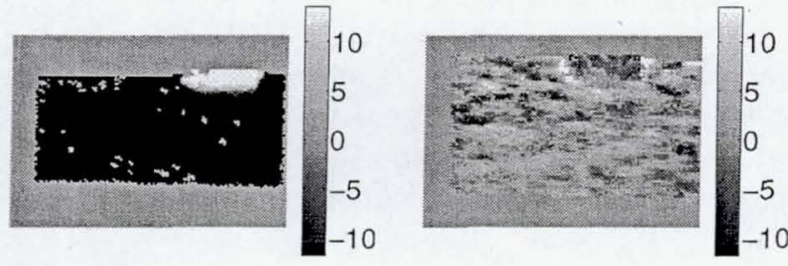

Figure 11: Depth error before and after registration for the pose estimation example. Error measured in centimeters. 

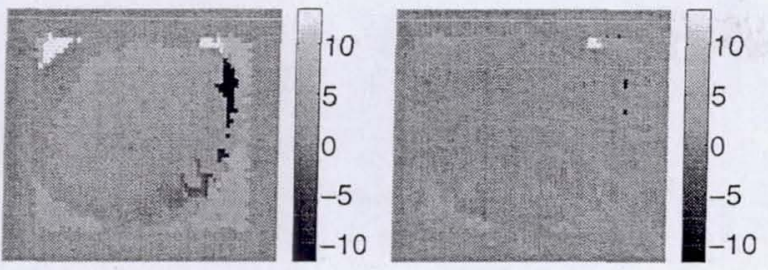

Figure 12: Depth error before and after registration for goal location hand off example. Errors measured in centimeters.

the Haz cam and Nav cam, the rover can hand off the target location from one view to the other. Figure 12 shows the depth errors before and after registration of surface models created with the Nav cam and Haz cam.

\section{Discussion}

Registration of 3D surface models is an attractive approach for rover localization. As long as the lighting conditions permit the acquisition of images for stereo, the resulting 3D surface models are independent of the lighting conditions. This is attractive compared to $2 \mathrm{D}$ approaches which might have difficulty with tracking features or recognizing places when lighting conditions change.

Furthermore, 2D visual tracking approaches require the rover to spend computational effort on something that it may only be doing for the purpose of visual odometry. However, NASA's current plans call for stereo vision to be used as a hazard avoidance technique on MER in 2003 and likely on MSL in 2009. Registering the 3D models that are already created in order to do local path planning and obstacle avoidance makes dual use of data that is being generated anyway. The marginal computation for registration is less than the computational effort for building the 3D surfaces, so most of the computational work is already done.

Accurate stereo calibration is fundamentally important for the approach in this paper. A miscalibration of stereo can cause the resulting models to be distorted by a projective transformation. Such a deformation will not be recoverable by the parameters in $\mathbf{p}$.

The robust estimation method used in this paper works quite well, as was reported in [13]. The surface models used in the examples here were not "cleaned" in any way and the results are still promising. Other reported approaches require mesh regularization and cleaning in order to ensure that there are no outliers before minimizing a norm which is sensitive to large deviations. These steps may improve the results we can acheive using robust estimation but empirically are not required for it to work.

We are currently working to further extend this work. Algorithmically we are investigating ways to optimize the implementation, perhaps making use of some very efficient existing rendering techniques. We would also like to extend this to multiview registration in order to handle more than two views at a time. It should be possible to extend the objective function to include a term which matches the albedo at each point along with the depth.

In terms of rover autonomy, this method might benefit from some sensor planning to determine when a next view is necessary for localization. We have already seen the difficulties with registering views where the rover has turned far enough that there is a small overlap between surface models.

This method is also being incorporated into a larger demonstration of single cycle instrument placement for improved efficiency of planetary rovers and increased science return for future Mars missions.

\section{Acknowledgements}

The authors would like to thank Larry Edwards, David Lees, Anne Wright, Susan Lee, and Maria Bualat for their help with the Viz visualization package, the K9 rover and the experiments in this paper. We would also like to thank Daniel Huber from Carnegie Mellon for many useful discussions on surface registration.

\section{References}

[1] L. Pedersen, R. Sargent, M. Bualat, M. Deans, C. Kunz, S. Lee, and A. Wright. Single cycle instrument deployment for mars rovers. To appear in Proceedings of $i$-SAIRAS, 2003. 
[2] Y. Chen and G. Medioni. Object modeling by registration of multiple range images. Image and Vision Computing, 10(3):145-155, 1992.

[3] P. J. Besl and N. D. McKay. A method for registration of 3-d shapes. IEEE Transactions on Pattern Analysis and Machine Intelligence, 14(2):239-256, 1992.

[4] B. K. P. Horn. Closed-form solution of absolute orientation using unit quatemions. Journal of the Optical Society of America, 4:629642, 1987.

[5] K. S. Arun, T. S. Huang, and S. D. Blostein. Least-squares fitting of two 3-d point sets. IEEE Transactions on Pattern Analysis and Machine Intelligence, PAMI-9(5):698700, 1987.

[6] A. J. Stoddart and A. Hilton. Registration of multiple point sets. In Proceedings of ICPR '96, pages 40-44, 1996.

[7] R. Benjemaa and F. Schmitt. A solution for the registration of multiple $3 \mathrm{~d}$ point sets using unit quaternions. In Proceedings of the European Conference on Computer Vision, volume 2, pages 34-50, 1998.

[8] A. Ardeshir Goshtasby. Three-dimensional model construction from multiview range images: survey with new results. IEEE Transactions on Pattern Analysis and Machine Intelligence, 31(11):1705-1714, 1998.

[9] Kari Pulli. Multiview registration for large data sets. In Int.Conf. on 3D Digital Imaging and Modeling, pages 160-168, 1999.

[10] S. Rusinkiewicz and M. Levoy. Efficient variants of the icp algorithm. In Third International Conference on $3 D$ Digital Imaging and Modeling (3DIM), 2001.

[11] P. Neugebauer. Geometrical cloning of 3d objects via simultaneous registration of multiple views. In Proceedings of the International Conference on Shape Modelling and Applications, pages 130-9, March 1997.

[12] J. Goldberger. Registration of multiple point sets using the em algorithm. In Proceedings of the International Conference on Computer Vision, volume 2, pages 730-736, 1999.

[13] A. Fitzgibbon. Robust registration of $2 \mathrm{~d}$ and 3d point sets. In British Machine Vision Conference, pages 411-420, 2001.

[14] W. Press, S. Teukolsky, W. Vetterling, and B. Flannery. Numerical Recipes in C. Cambridge University Press, 1988.

[15] Philip E. Gill, Walter Murray, and Margaret H. Wright. Practical Optimization. Academic Press, 1981.

[16] P. J. Huber. Robust Statistics. John Wiley \& Sons, 1981.

[17] Kenneth Levenberg. A method for the solution of certain non-linear problems in least-squares. Quarterly of Applied Mathematics, 2(2):164168, July 1944.

[18] Donald W. Marquardt. An algorithm for the least-squares estimation of nonlinear parameters. SIAM Journal of Applied Mathematics, 11(2):431-441, June 1963.

[19] William. J. J. Rey. Introduction to Robust and Quasi-Robust Statistical Methods. Springer, 1983.

[20] Zhengyou Zhang. Parameter estimation techniques: A tutorial with application to conic fitting. Technical Report No. 2676, INRIA, 1995.

[21] L. Nguyen et al. Virtual reality interfaces for visualization and control of remote vehicles. Autonomous Robots, 11(1), 2001.

[22] J. Bresina, M. Bualat, L. Edwards, R. Washington, and $\mathrm{A}$. Wright. K9 operations in may '00 dual-rover field experiment. In $i$-SAIRAS, 2001. 